1999-7

\title{
Successive Galerkin Approximation of the Isaacs Equation
}

Timothy McLain

Mechanical Engineering Department, Brigham Young University, mclain@byu.edu

Randal W. Beard

Department of Electrical and Computer Engineering, Brigham Young University, beard@ee.byu.edu

John T. Wen

Electrical, Computer and Systems Engineering Rensselaer Polytechnic Institute

Follow this and additional works at: https://scholarsarchive.byu.edu/facpub

Part of the Mechanical Engineering Commons

\section{Original Publication Citation}

Beard, R., McLain, T., and Wen, J. Successive Galerkin Approximation of the Isaacs Equation, Proceedings of the 14th IFAC World Congress, no. 5, pp. 717-743, July 1999, Beijing, China.

\section{BYU ScholarsArchive Citation}

McLain, Timothy; Beard, Randal W.; and Wen, John T., "Successive Galerkin Approximation of the Isaacs Equation" (1999). Faculty Publications. 1938.

https://scholarsarchive. byu.edu/facpub/1938

This Conference Paper is brought to you for free and open access by BYU ScholarsArchive. It has been accepted for inclusion in Faculty Publications by an authorized administrator of BYU ScholarsArchive. For more information, please contact ellen_amatangelo@byu.edu. 


\title{
SUCCESSIVE GALERKIN APPROXIMATION OF THE ISAACS EQUATION.
}

\author{
Randal W. Beard ${ }^{*, 1}$ Timothy W. McLain ${ }^{* *}$ John T. Wen*** \\ * Electrical and Computer Engineering, Brigham Young University, \\ beard@ee.byu.edu \\ ${ }^{* *}$ Mechanical Engineering, Brigham Young University \\ *** Electrical, Computer and Systems Engineering, Rensselaer Polytechnic Institute
}

\begin{abstract}
The successive Galerkin approximation (SGA) algorithm has recently been developed for approximating solutions to the Hamilton-Jacobi-Isaacs equation. The algorithm produces feedback control laws that are stabilizing on a well-defined region of state space. The objective of this paper is to demonstrate the application of the SGA algorithm to two simple examples. The examples serve several purposes: first they illustrate how the algorithm is applied in a setting that is simple enough to write out in detail, second they demonstrate the convergence of the algorithm in a setting where the actual solution can be derived analytically.
\end{abstract}

Keywords. $\mathcal{H}_{\infty}$ control, nonlinear control, partial differential equations, numerical algorithms, successive approximation, Galerkin approximation.

\section{INTRODUCTION}

Linear $\mathcal{H}_{\infty}$ control has recently been extended to nonlinear systems (van der Schaft, 1992; Isidori and Astolfi, 1992; Ball et al., 1993). Although the theory for nonlinear $\mathcal{H}_{\infty}$ control is well developed, there have been very few applications, due to the lack of efficient numerical methods. To solve the nonlinear, state-feedback $\mathcal{H}_{\infty}$ control problem requires the solution of the HamiltonJacobi-Isaacs (HJI) equation, which is a first order, nonlinear partial differential equation, that has proven to be impossible to solve in general, and very difficult to solve for specific nonlinear systems. Since this equation is difficult to solve analytically, there has been a great deal of research directed toward approximating its solution (Wise and Sedwick, 1994; Huang and Lin, 1995; Fleming and Soner, 1993).

\footnotetext{
1 Supported in part by the National Science Foundation under Grant ESC-9732917
}

Recently, we have developed an approximation algorithm, called the Successive Galerkin Approximation (SGA) algorithm, for the Hamilton-Jacobi-Bellman (HJB) equation that has several desirable characteristics, namely (1) feedback control, (2) guaranteed stability, (3) a welldefined region of state-space over which the approximation is valid, and (4) off-line computations (Beard, 1995; Beard et al., 1996; Beard et al., 1997; Beard et al., 1998). In (Beard and McLain, 1998) this algorithm was extended to the Hamilton-Jacobi-Isaacs equation, however the convergence properties of the extended algorithm have not yet been studied in detail.

The objective of this paper is to describe the SGA algorithm for the HJI equation and to demonstrate through two academic examples with known solutions to the HJI Equation, that the approximate solution converges to the solution of the HJI equation.

The remainder of the paper is organized as follows. In 
Section 2, the successive Galerkin approximation (SGA) algorithm for the HJI equation is stated. Section 3 states what is currently known mathematically about the convergence of the algorithm. Section 4 demonstrates the convergence for two simple examples. Finally, Section 5 gives our conclusions.

\section{THE ALGORITHM}

This section gives a brief overview of the SGA algorithm applied to the HJI equation. See (Beard and McLain, 1998) for greater detail.

The nonlinear $\mathcal{H}_{\infty}$ control problem is stated in terms of the $\mathcal{L}_{2}$ gain of a system. Consider the system

$$
\begin{aligned}
& \dot{x}=f(x)+g(x) u+k(x) w \\
& y=h(x),
\end{aligned}
$$

where $x \in \mathrm{R}^{n}$ is the state, $u \in \mathrm{R}^{m}$ is the control, $w \in$ $\mathrm{R}^{q}$ is the disturbance, $y \in \mathrm{R}^{p}$ is the output and where $h(0)=0$ and $f$ is observable through $h$.

System (1) is said to have $\mathcal{L}_{2}$ gain less than or equal to $\gamma$ if for all $T \geq 0$ and $w \in \mathcal{L}_{2}(0, T)$,

$$
\int_{0}^{T}\left(\|y(t)\|^{2}+\|u(t)\|_{R}^{2}\right) d t \leq \gamma^{2} \int_{0}^{T}\|w(t)\|_{P}^{2} d t
$$

where $x(0)=0$. System (1) has $\mathcal{L}_{2}$-gain less than $\gamma$ if there exists some $0 \leq \tilde{\gamma} \leq \gamma$ such that equation (2) holds for $\tilde{\gamma}$.

The nonlinear $\mathcal{H}_{\infty}$ (state-feedback) optimal control problem is to find, if it exists, the smallest value $\gamma^{*} \geq 0$ and an associated state feedback control law $u_{\gamma}^{*}(x)$ such that for any $\gamma>\gamma^{*}$, system (1) under the control of $u_{\gamma}^{*}$ has $\mathcal{L}_{2}$-gain less than or equal to $\gamma$. As van der Schaft (van der Schaft, 1992) shows, a sufficient condition for system (1) to have $\mathcal{L}_{2}$-gain less than or equal to $\gamma>0$ is that

$$
u_{\gamma}(x)=-\frac{1}{2} R^{-1} g^{T}(x) \frac{\partial V_{\gamma}}{\partial x},
$$

where $V_{\gamma} \geq 0$ is a smooth solution to the HJI equation

$$
\begin{aligned}
& \frac{\partial V_{\gamma}^{T}}{\partial x} f+h^{T} h \\
& +\frac{1}{4} \frac{\partial V_{\gamma}^{T}}{\partial x}\left(\frac{1}{2 \gamma^{2}} k P^{-1} k^{T}-g R^{-1} g^{T}\right) \frac{\partial V_{\gamma}}{\partial x}=0,
\end{aligned}
$$

with the boundary condition $V_{\gamma}(0)=0$.

Two algorithms will now be stated: the successive approximation (SA) algorithm, and the successive Galerkin approximation (SGA) algorithm to the HJI equation. Since the derivation of the SA and SGA algorithms for the HJI equation appear in (Beard and McLain, 1998), they will not be derived here, only the main results will be stated. The SA algorithm is a duel iteration in policy space that reduces the HJI equation to an infinite sequence of nonlinear partial differential equations.

\section{Algorithm 1 (SA)}

Let $u^{(0)}$ be an initial stabilizing control law for the system $\dot{x}=f+g u(w \equiv 0)$, with stability region $\Omega$.

For $i=0$ to $\infty$

Set $w_{\gamma}^{(i, 0)} \equiv 0$.

For $j=0$ to $\infty$

Solve for $V_{\gamma}^{(i, j)}$ from:

$$
\begin{aligned}
\frac{\partial V_{\gamma}^{(i, j) T}}{\partial x}(f & \left.+g u_{\gamma}^{(i)}+k w_{\gamma}^{(i, j)}\right)+h^{T} h \\
& +\left\|u_{\gamma}^{(i)}\right\|_{R}^{2}-\gamma^{2}\left\|w_{\gamma}^{(i, j)}\right\|_{R}^{2}=0 .
\end{aligned}
$$

Update the Disturbance:

$$
w_{\gamma}^{(i, j+1)}=\frac{1}{2 \gamma^{2}} P^{-1} k^{T} \frac{\partial V_{\gamma}^{(i, j)}}{\partial x} .
$$

\section{End $j$ Loop}

\section{Update the Control:}

$$
u_{\gamma}^{(i+1)}=-\frac{1}{2} R^{-1} g^{T} \frac{\partial V_{\gamma}^{(i, \infty)}}{\partial x} .
$$

\section{End $i$ Loop}

The difficulty with Algorithm 1 is that Equation (4) is also difficult to solve. To approximation this equation, we employ a global Galerkin approximation to obtain the following algorithm.

\section{Algorithm 2 (SGA)}

Let $u^{(0)}$ be an initial stabilizing control law for the system $\dot{x}=f+g u$ with stability region $\Omega$.

\section{Pre-compute the integrals}

$$
\begin{aligned}
\mathbf{A}_{1} & \triangleq \int_{\Omega} \Phi_{N} f^{T} \nabla \Phi_{N}^{T} d x \\
\mathbf{A}_{2}\left(u^{(0)}\right) & \triangleq \int_{\Omega} \Phi_{N} u^{(0) T} g^{T} \nabla \Phi_{N}^{T} d x \\
\mathbf{b}_{1} & \triangleq-\int_{\Omega} \Phi_{N} h^{T} h d x \\
\mathbf{b}_{2}\left(u^{(0)}\right) & \triangleq-\int_{\Omega} \Phi_{N}\left\|u^{(0)}\right\|_{R}^{2} d x \\
\mathbf{G}_{j} & \triangleq \int_{\Omega} \Phi_{N} \frac{\partial \phi_{j}^{T}}{\partial x} g R^{-1} g^{T} \nabla \Phi_{N}^{T} d x, \quad j=1, \ldots, N \\
\mathbf{K}_{j} & \triangleq \int_{\Omega} \Phi_{N} \frac{\partial \phi_{j}^{T}}{\partial x} k P^{-1} k^{T} \nabla \Phi_{N}^{T} d x, \quad j=1, \ldots, N .
\end{aligned}
$$

While $\left\|\mathbf{c}_{\gamma, N}^{(i+1, j)}-\mathbf{c}_{\gamma, N}^{(i, j)}\right\|>\epsilon$

Let 


$$
\begin{aligned}
& \mathbf{A}^{(i)}= \begin{cases}\mathbf{A}_{1}+\mathbf{A}_{2}\left(u^{(0)}\right) & i=0 \\
\mathbf{A}_{1}-\frac{1}{2} \sum_{k=1}^{\infty} c_{k}^{(i-1, \infty)} \mathbf{G}_{k} & i>0\end{cases} \\
& \mathbf{b}^{(i)}= \begin{cases}\mathbf{b}_{1}+\mathbf{b}_{2}\left(u^{(0)}\right) & i=0 \\
\mathbf{b}_{1}-\frac{1}{4} \sum_{k=1}^{\infty} c_{k}^{(i-1, \infty)} \mathbf{G}_{k} \mathbf{c}_{N}^{(i-1, \infty)} & i>0\end{cases}
\end{aligned}
$$

While $\left\|\mathbf{c}_{\gamma, N}^{(i, j+1)}-\mathbf{c}_{\gamma, N}^{(i, j)}\right\|>\epsilon$

$$
\begin{aligned}
& \mathbf{A}= \begin{cases}\mathbf{A}^{(i)} & j=0 \\
\mathbf{A}^{(i)}+\frac{1}{2 \gamma^{2}} \sum_{k=1}^{\infty} c_{k}^{(i, j-1)} \mathbf{G}_{k} & j>0\end{cases} \\
& \mathbf{b}= \begin{cases}\mathbf{b}^{(i)} & j=0 \\
\mathbf{b}^{(i)}+\frac{1}{4 \gamma^{2}} \sum_{k=1}^{\infty} c_{k}^{(i, j-1)} \mathbf{G}_{k} \mathbf{c}_{N}^{(i, j-1)} & j>0\end{cases}
\end{aligned}
$$

Solve for $V_{\gamma, N}^{(i, j)}$ from:

Update the Disturbance:

$$
\mathbf{c}_{N}^{(i, j)}=\mathbf{A}^{-1} \mathbf{b}
$$

$$
w_{\gamma, N}^{(i, j+1)}=\frac{1}{2 \gamma^{2}} P^{-1} k^{T} \nabla \Phi_{N}^{T} \mathbf{c}_{N}^{(i, j)}
$$

$$
j=j+1
$$

\section{End $j$ Loop}

\section{Update the Control:}

$$
u_{\gamma, N}^{(i+1)}=-\frac{1}{2} R^{-1} g^{T} \nabla \Phi_{N}^{T} \mathbf{c}_{N}^{(i, \infty)}
$$

$$
i=i+1
$$

End $i$ Loop

\section{CONVERGENCE ANALYSIS}

The following convergence results for Algorithm 1 are proved in (Beard and McLain, 1998).

Lemma 1 If for each $(i, j)$ the system

$$
\dot{x}=f+g u_{\gamma}^{(i)}+k w_{\gamma}^{(i, j)}
$$

is asymptotically stable on $\Omega$, then

$$
V_{\gamma}^{(i, j)}(x) \leq V_{\gamma}^{(i, j+1)}(x) \leq V_{\gamma}^{(i, \infty)}(x)
$$

for each $x \in \Omega$, where $V_{\gamma}^{(i, \infty)}$ solves the equation

$$
\begin{aligned}
\frac{\partial V_{\gamma}^{(i, \infty)}}{\partial x}(f & \left.+g u_{\gamma}^{(i)}\right)+h^{T} h+\left\|u_{\gamma}^{(i)}\right\|_{R}^{2} \\
& +\frac{1}{4 \gamma^{2}} \frac{\partial V_{\gamma}^{(i, \infty)}}{\partial x} k P^{-1} k^{T} \frac{\partial V_{\gamma}^{(i, \infty)}}{\partial x}=0 .
\end{aligned}
$$

Furthermore, if $V_{\gamma}^{(i, j+1)}(x)=V_{\gamma}^{(i, j)}(x)$, then $V_{\gamma}^{(i, j+1)}(x)=V_{\gamma}^{(i, \infty)}(x)$, i.e., $V_{\gamma}^{(i, j)} \rightarrow V_{\gamma}^{(i, \infty)}$ pointwise on $\Omega$.
Lemma 2 If for each $i$ the system

$$
\dot{x}=f+g u_{\gamma}^{(i)}+k w_{\gamma}^{(i, \infty)}
$$

is asymptotically stable on $\Omega$, then

$$
V_{\gamma}^{(i, \infty)}(x) \geq V_{\gamma}^{(i+1, \infty)}(x) \geq V_{\gamma}
$$

for each $x \in \Omega$, where $V_{\gamma}$ solves the HJI equation (3).

Furthermore, if $V_{\gamma}^{(i+1, \infty)}(x)=V_{\gamma}^{(i, \infty)}(x)$, then $V_{\gamma}^{(i+1, \infty)}(x)=V_{\gamma}(x)$, i.e., $V_{\gamma}^{(i, \infty)} \rightarrow V_{\gamma}$ pointwise on $\Omega$.

Lemma 3 If $u_{\gamma}^{(i)}(x)$ is asymptotically stabilizing on $\Omega$, then so is $u_{\gamma}^{(i+1)}(x)$. Furthermore $V_{\gamma}^{(i, \infty)}$ is a Lyapunov function for $u_{\gamma}^{(i+1)}$.

\section{TWO SIMPLE EXAMPLES}

In this section we will demonstrate that Algorithm 2 in fact converges for two academic examples. The first example is for a linear system, where the solution to the HJI equation is $V_{\gamma}(x)=x^{T} Q x$ where $Q=Q^{T}$ is the solution to the following Matrix Algebraic Riccati Equation

$$
\begin{aligned}
Q F+F^{T} Q+H^{T} H- & Q G^{T} R^{-1} G Q \\
& +\frac{1}{\gamma^{2}} Q K P^{-1} K^{T} Q=0 .
\end{aligned}
$$

For the standard optimal control problem, the successive approximation algorithm leads to the well-known Kleinman algorithm for solving the standard Algebraic Riccati Equation from optimal control theory (Kleinman, 1968). By substituting $V_{\gamma}^{(i, j)}=x^{T} Q^{(i, j)} x$ into Algorithm 1 we obtain the following algorithm which can be considered as an extension of the Kleinman algorithm to Equation (11).

\section{Algorithm 3 (Kleinman)}

Let $u^{(0)}=-L^{(0)} x$ be an initial asymptotically stabilizing control law for the system $\dot{x}=F x+G u$.

While $\left\|L^{(i+1)}-L^{(i)}\right\|>\epsilon$ and $i<I_{\max }$,

Set $S^{(i, 0)} \equiv \mathbf{0}$.

While $\left\|S^{(i, j+1)}-S^{(i, j)}\right\|>\epsilon$ and $j<J_{\max }$,

Solve for $Q^{(i, j)}$ from:

$$
\begin{aligned}
& Q^{(i, j)}\left[F+G L^{(i)}+K S^{(i, j)}\right] \\
& +\left[F+G L^{(i)}+K S^{(i, j)}\right]^{T} Q^{(i, j)} \\
& +H^{T} H+L^{(i)} R L^{(i)} \\
& \quad-\gamma^{2} S^{(i, j)} P S^{(i, j)}=0
\end{aligned}
$$

\section{Update the Disturbance:}




$$
j=j+1
$$

$$
S^{(i, j+1)}=\frac{1}{\gamma^{2}} P^{-1} K^{T} Q^{(i, j)} .
$$

\section{End $j$ Loop}

\section{Update the Control:}

$i=i+1$

$$
L^{(i+1)}=-R^{-1} G^{T} Q^{(i, j)} .
$$

\section{End $i$ Loop}

Using the results in Section 3 it is straight forward to show that Algorithm 3 converges to the solution of Equation (11) (for large enough $\gamma$ ). In the next subsection we will show that for linear systems, Algorithms 2 and 3 produce the same results.

To this point, the role of the parameter $\gamma$ has been ignored. It is known that there exists a $\gamma^{*}$ such that if $\gamma \leq \gamma^{*}$, then there is no positive definite solution to Equation (3), and if $\gamma>\gamma^{*}$, then there exist a unique positive definite solution to Equation (3) (Basar and Bernhard, 1995). We have observed that Algorithm 2 converges for $\gamma \geq \gamma_{\min }$ and does not converge otherwise. We conjecture that as the order of approximation increases, $\gamma_{\text {min }} \rightarrow \gamma^{*}$. However, this remains to be shown. In the following examples, $\gamma_{\min }$ is found by using the following binary search algorithm, assuming that the input/output structure of the SGA/Kleinman algorithm is given as

$$
\begin{aligned}
{\left[\mathbf{c}^{(i, j)}, i, j\right] } & =S G A\left(\mathbf{c}^{(0,0)}, \gamma\right) \\
{\left[Q^{(i, j)}, i, j\right] } & =K S A\left(Q^{(0,0)}, \gamma\right) .
\end{aligned}
$$

\section{Algorithm 4 (Gamma)}

\section{Initialize:}

$\gamma_{L}=0, \gamma_{U}=1, i=I_{\max }, j=J_{\max }$.

\section{Find Upper Bound for $\gamma$}

While $i \geq I_{\max }$ or $j \geq J_{\max }$,

$$
\begin{aligned}
& \gamma_{U}=2 \gamma_{U} \\
& {\left[\mathbf{c}^{(i, j)}, i, j\right]=S G A\left(\mathbf{c}^{(0,0)}, \gamma_{U}\right)}
\end{aligned}
$$

Find $\gamma_{\min }$

While $\left|\gamma_{U}-\gamma_{L}\right|>\delta$

$$
\begin{aligned}
& \gamma_{\min }=\frac{\gamma_{U}+\gamma_{L}}{2} \\
& {\left[\mathbf{c}^{(i, j)}, i, j\right]=S G A\left(\mathbf{c}^{(0,0)}, \gamma_{\min }\right)} \\
& \text { If } i<I_{\max } \text { and } j<J_{\max } \\
& \text { Then } \gamma_{U}=\gamma_{\min } \\
& \text { Else } \gamma_{L}=\gamma_{\min }
\end{aligned}
$$

\subsection{Example: Linear Single-State System}

The first example is the simple, first order linear system

$$
\begin{aligned}
& \dot{x}=-2 x+u+w \\
& y=x,
\end{aligned}
$$

where $R=P=1$. According to (Green and Limebeer, 1995 , p. 240), for this system $\gamma^{*}=0.4472$, and $V_{\gamma}=$ $Q^{*} x^{2}$ where

$$
Q^{*}=0.4084
$$

A stabilizing control for this system is $u=-2 x$, therefore let $L^{(0)}=-2$. Letting $\gamma=1$, Algorithm 3 is applied as follows:

$$
\begin{aligned}
Q^{(i, j)} & =\frac{-1-L^{(i) 2}+\gamma^{2} S^{(i, j) 2}}{2\left(-2+L^{(i)}+S^{(i, j)}\right)} \\
S^{(i, j)} & =\frac{1}{\gamma^{2}} Q^{(i, j)} \\
L^{(i)} & =-Q^{(i, j)} .
\end{aligned}
$$

The following table shows the values for $Q, S$, and $L$ as functions of $i, j$.

\begin{tabular}{c|ccc}
$(i, j)$ & $Q^{(i, j)}$ & $S^{(i, j)}$ & $L^{(i)}$ \\
\hline$(0,0)$ & 0.6250 & 0.0000 & -2.0000 \\
$(0,1)$ & 0.6829 & 0.6250 & -2.0000 \\
$(0,2)$ & 0.6834 & 0.6829 & -2.0000 \\
$(0,3)$ & 0.6844 & 0.6834 & -2.0000 \\
\hline$(1,0)$ & 0.2734 & 0.0000 & -0.6834 \\
$(1,1)$ & 0.2889 & 0.2734 & -0.6834 \\
$(1,2)$ & 0.2889 & 0.2889 & -0.6834 \\
\hline$(2,0)$ & 0.2367 & 0.0000 & -0.2889 \\
$(2,1)$ & 0.2503 & 0.2367 & -0.2889 \\
$(2,2)$ & 0.2504 & 0.2503 & -0.2889 \\
\hline$(3,0)$ & 0.2361 & 0.0000 & -0.2504 \\
$(3,1)$ & 0.2500 & 0.2361 & -0.2504 \\
$(3,2)$ & 0.2500 & 0.2500 & -0.2504
\end{tabular}

If the algorithm is embedded in Algorithm 4, then the final results are $\gamma=0.4590, Q=0.3996, S=0.3996$, and $L=-0.3996$. Note that the values for $Q$ increase with $j$ for each fixed $i$ and decrease with iterations in $i$. This is a general trait of the algorithm.

The application of Algorithm 2 to the same system will now be described. Again, let the initial stabilizing control be $u=-2 x$, and let $\Omega=[-1,1]$. Let there be one basis element, namely $\phi_{1}(x)=x^{2}$. Then from Algorithm 2 ,

$$
\begin{aligned}
\mathbf{A}_{1} & =\int_{-1}^{1}\left(x^{2}\right)(-2 x)(2 x) d x=-1.6 \\
\mathbf{A}_{2}\left(u^{(0)}\right) & =\int_{-1}^{1}\left(x^{2}\right)(-2 x)(2 x) d x=-1.6 \\
\mathbf{b}_{1} & =-\int_{-1}^{1}\left(x^{2}\right) d x=-0.6667 \\
\mathbf{b}_{2}\left(u^{(0)}\right) & =-\int_{-1}^{1}\left(x^{2}\right)\left(4 x^{2}\right) d x=1.6 \\
\mathbf{G}_{1} & =\int_{-1}^{1}\left(x^{2}\right)(2 x)(2 x) d x=1.6
\end{aligned}
$$




$$
\mathbf{K}_{1}=\int_{-1}^{1}\left(x^{2}\right)(2 x)(2 x) d x=1.6
$$

The following table shows the values for $\mathbf{c}_{\gamma N}^{(i, j)}, \mathbf{w}_{\gamma, N}^{(i, j)}$ and $\mathbf{u}_{\gamma, N}^{(i)}$ that result from the application of Algorithm 2 when $\gamma=1$.

\begin{tabular}{c|ccc}
$(i, j)$ & $\mathbf{c}_{\gamma, N}^{(i, j)}$ & $\mathbf{w}_{\gamma, N}^{(i, j)}$ & $\mathbf{u}_{\gamma, N}^{(i)}$ \\
\hline$(0,0)$ & 0.6250 & $0.0000 x$ & $-2.0000 x$ \\
$(0,1)$ & 0.6829 & $0.6250 x$ & $-2.0000 x$ \\
$(0,2)$ & 0.6834 & $0.6829 x$ & $-2.0000 x$ \\
\hline$(1,0)$ & 0.2500 & $0.0000 x$ & $-0.6834 x$ \\
$(1,1)$ & 0.2886 & $0.2500 x$ & $-0.6834 x$ \\
$(1,2)$ & 0.2889 & $0.2886 x$ & $-0.6834 x$ \\
$(1,3)$ & 0.2890 & $0.2889 x$ & $-0.6834 x$ \\
\hline$(2,0)$ & 0.2500 & $0.0000 x$ & $-0.2890 x$ \\
$(2,1)$ & 0.2504 & $0.2500 x$ & $-0.2890 x$ \\
$(2,2)$ & 0.2504 & $0.2504 x$ & $-0.2890 x$ \\
\hline$(3,0)$ & 0.2500 & $0.0000 x$ & $-0.2504 x$ \\
$(3,1)$ & 0.2500 & $0.2500 x$ & $-0.2504 x$
\end{tabular}

Note the almost identical agreement with the values in the previous table. When embedded in algorithm 4 , the algorithm converges to the following values: $\gamma=0.4472$, $V_{\gamma}=0.4084 x^{2}, \mathbf{w}=1.9598 x$, and $\mathbf{u}=-0.4084 x$. Recall that the true optimal value of $\gamma$ is 0.4472 and the optimal value of $V_{\gamma}$ is $0.4084 x^{2}$, showing that Algorithm 2 in conjunction with Algorithm 4 converge to the correct values for this example.

\subsection{Example: Nonlinear Single-State System}

For the second example, we will look at the nonlinear system given by

$$
\begin{aligned}
& \dot{x}=x-x^{3}+u+w \\
& y=x,
\end{aligned}
$$

with $R=P=1$, and again $\Omega=[-1,1]$. For this system the HJI equation is

$$
\frac{\partial V_{\gamma}}{\partial x}\left(x-x^{3}\right)+x^{2}+\frac{1}{4}\left(\frac{1}{2 \gamma^{2}}-1\right) \frac{\partial V_{\gamma}^{2}}{\partial x}=0,
$$

which is a quadratic function in $\frac{\partial V_{\gamma}}{\partial x}$. Defining $\epsilon \triangleq \frac{1}{4}(1-$ $\left.\frac{1}{2 \gamma^{2}}\right)$, solving for $\frac{\partial V_{\gamma}}{\partial x}$, and selecting the stabilizing solution, we obtain

$$
\frac{\partial V_{\gamma}}{\partial x}=\frac{\left(x-x^{3}\right)+x \sqrt{\left(x^{2}-1\right)^{2}+4 \epsilon}}{2 \epsilon}
$$

which can be integrated over the region $\Omega=[-1,1]$ with boundary condition $V_{\gamma}(0)=0$ to obtain the true value for $V_{\gamma}^{*}(x)$. The plot of $V_{\gamma}^{*}$ for $\gamma=10$ is depicted in Figure 1 by a solid line.

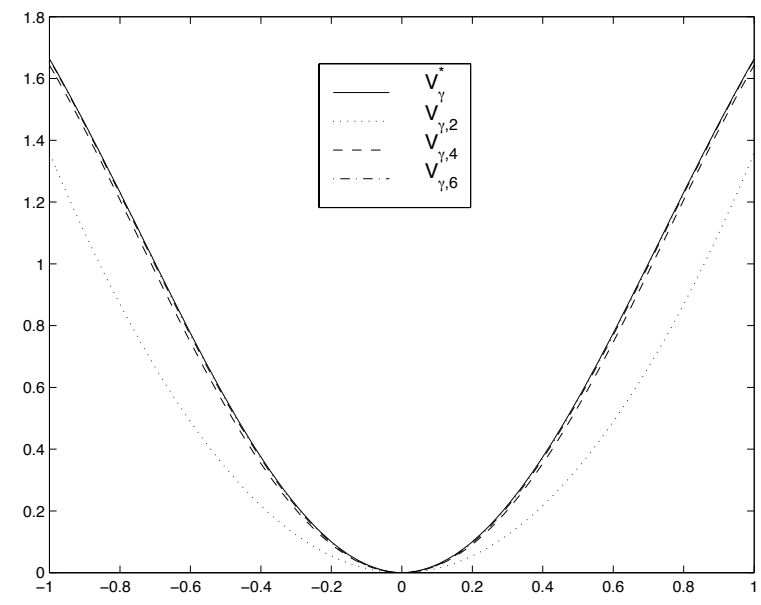

Fig. 1. Plots of the true and estimated value function.

We will now demonstrate the application of Algorithm 2 to this problem. Letting the basis functions be $\left\{x^{2}, x^{4}\right\}$, and the initial stabilizing control be $u=-2 x+x^{3}$, we obtain the following values for the integrals in the initialization step of Algorithm 2:

$\mathbf{A}_{1}$

$$
\begin{aligned}
& =\left(\begin{array}{l}
\int_{-1}^{1}\left(x^{2}\right)\left(x-x^{3}\right)(2 x) d x, \int_{-1}^{1}\left(x^{2}\right)\left(x-x^{3}\right)\left(4 x^{3}\right) d x \\
\int_{-1}^{1}\left(x^{4}\right)\left(x-x^{3}\right)(2 x) d x, \int_{-1}^{1}\left(x^{4}\right)\left(x-x^{3}\right)\left(4 x^{3}\right) d x
\end{array}\right) \\
& =\left(\begin{array}{l}
0.2286,0.2540 \\
0.1270,0.1616
\end{array}\right)
\end{aligned}
$$

$$
\mathbf{A}_{2}\left(u^{(0)}\right)
$$$$
=\left(\begin{array}{l}
\int_{-1}^{1}\left(x^{2}\right)\left(-2 x+x^{3}\right)(2 x) d x, \int_{-1}^{1}\left(x^{2}\right)\left(-2 x+x^{3}\right)\left(4 x^{3}\right) d x \\
\int_{-1}^{1}\left(x^{4}\right)\left(-2 x+x^{3}\right)(2 x) d x, \int_{-1}^{1}\left(x^{4}\right)\left(-2 x+x^{3}\right)\left(4 x^{3}\right) d x
\end{array}\right)
$$$$
=\left(\begin{array}{l}
-1.0286,-1.3968 \\
-0.6984,-1.0505
\end{array}\right)
$$

$\mathbf{b}_{1}=\left(\int_{-1}^{1}\left(x^{2}\right)\left(x^{2}\right) d x, \int_{-1}^{1}\left(x^{4}\right)\left(x^{2}\right) d x\right)^{T}=(0.4000,0.2857)^{T}$

$\mathbf{b}_{2}\left(u^{(0)}\right)=\left(\int_{-1}^{1}\left(x^{2}\right)\left(-2 x+x^{3}\right)^{2} d x, \int_{-1}^{1}\left(x^{4}\right)\left(-2 x+x^{3}\right)^{2} d x\right)^{T}$ $=(0.6794,0.4358)^{T}$

$\mathbf{G}_{1}=\left(\begin{array}{l}\int_{-1}^{1}\left(x^{2}\right)(2 x)(2 x) d x, \int_{-1}^{1}\left(x^{2}\right)(2 x)\left(4 x^{3}\right) d x \\ \int_{-1}^{1}\left(x^{4}\right)(2 x)(2 x) d x, \int_{-1}^{1}\left(x^{4}\right)(2 x)\left(4 x^{3}\right) d x\end{array}\right)$

$=\left(\begin{array}{l}1.6000,2.2857 \\ 1.1429,1.7778\end{array}\right)$ 


$$
\begin{aligned}
& \mathbf{G}_{2}=\left(\begin{array}{l}
\int_{-1}^{1}\left(x^{2}\right)\left(4 x^{3}\right)(2 x) d x, \int_{-1}^{1}\left(x^{2}\right)\left(4 x^{3}\right)\left(4 x^{3}\right) d x \\
\int_{-1}^{1}\left(x^{4}\right)\left(4 x^{3}\right)(2 x) d x, \int_{-1}^{1}\left(x^{4}\right)\left(4 x^{3}\right)\left(4 x^{3}\right) d x
\end{array}\right) \\
& =\left(\begin{array}{c}
2.2857,3.5556 \\
1.7778,2.9091
\end{array}\right) \\
& \mathbf{K}_{j}=\mathbf{G}_{j}, \quad j=1, \ldots, N .
\end{aligned}
$$

The application of Algorithm 2 results in

$$
\mathbf{c}_{10,4}=(2.3180,-0.6750)^{T}
$$

which implies that $V_{10,4}(x)=2.3180 x^{2}-0.6750 x^{4}$ which is depicted in Figure 1 by a dashed line. Also plotted in Figure 1 are $V_{10,2}$ and $V_{10,6}$. It is clear from the figure that $V_{\gamma, N}$ is converging to $V_{\gamma}^{*}$ on $\Omega$.

\section{CONCLUSIONS}

The objective of this paper is to demonstrate the application of the successive Galerkin approximation algorithm to the Hamilton-Jacobi-Isaacs equation. In particular, the algorithms was applied to two first order systems. Several conclusions can be drawn from these examples. First, it appears that the SGA algorithm converges to the optimal value function for a fixed $\gamma$. The convergence is interesting in that there is an inner and an outer loop. The value function uniformly increases in the inner loop, and uniformly decreases in the outer loop. There is a game theoretic interpretation to this iteration. In the inner loop, the control is fixed and the disturbance it iteratively "improved," in the sense that the value function is increasing. Once the disturbance is made as large as possible, without destroying the stability of the system, then one application of the outer loop improves the control for that particular disturbance.

Further research is still needed to fully understand the convergence properties of Algorithm 2.

\section{REFERENCES}

Ball, Joseph A., J. William Helton and Michael L. Walker (1993). $H_{\infty}$ control for nonlinear systems with output feedback. IEEE Transactions on Automatic Control 38(4), 548-559.

Basar, Tamer and Pierre Bernhard (1995). $H_{\infty}$-Optimal Control and Related Minimax Design Problems. Birkhaüser.

Beard, Randal (1995). Improving the Closed-Loop Performance of Nonlinear Systems. PhD thesis. Rensselaer Polytechnic Institute. Troy, New York.
Beard, Randal, George Saridis and John Wen (1996). Improving the performance of stabilizing control for nonlinear systems. Control Systems Magazine 16(5), 27-35.

Beard, Randal, George Saridis and John Wen (1997). Galerkin approximation of the generalized Hamilton-Jacobi-Bellman equation. Automatica 33(12), 2159-2177.

Beard, Randal, George Saridis and John Wen (1998). Approximate solutions to the time-invariant Hamilton-Jacobi-Bellman equation. Journal of $O p$ timization Theory and Applications.

Beard, Randal W. and Timothy W. McLain (1998). Successive Galerkin approximation algorithms for nonlinear optimal and robust control. International Journal of Control 71(5), 717-743.

Fleming, Wendell H. and H. Mete Soner (1993). Controlled Markov Processes and Viscosity Solutions. Springer Verlag. Berlin, Germany.

Green, Michael and David J. N. Limebeer (1995). Linear Robust Control. Prentice Hall.

Huang, Jie and Ching-Fang Lin (1995). Numerical approach to computing nonlinear $h_{\infty}$ control laws. AIAA Journal of Guidance, Control and Dynamics 18(5), 989-994.

Isidori, Alberto and Alessandro Astolfi (1992). Disturbance attenuation and $H_{\infty}$-control via measurement feedback in nonlinear systems. IEEE Transactions on Automatic Control 37(9), 1283-1293.

Kleinman, David L. (1968). On an iterative technique for Riccati equation computations. IEEE Transactions on Automatic Control 13, 114-115.

van der Schaft, A. J. (1992). $L_{2}$-gain analysis of nonlinear systems and nonlinear state feedback $H_{\infty}$ control. IEEE Transactions on Automatic Control 37(6), 770-784.

Wise, Kevin A. and Jack L. Sedwick (1994). Succesive approximation solution of the HJI equation. In: Proceedings of the 33rd Conference on Decision and Control. Lake Buena Vista, FL. pp. 1387-1391. 